\title{
Comparação entre os diagnósticos clínicos e os achados de necropsia: análise retrospectiva de 680 pacientes
}

\section{Correlation between clinical and autopsy diagnoses: a retrospective analysis of 680 patients}

Marcos Emanuel de Alcântara Segura' ${ }^{1}$ Erudith Mendes Rocha' ${ }^{1}$, Alexandre Alves Lourenço', Moema Gonçalves Pinheiro Veloso ${ }^{2}$, Waldete Cabral Moraes ${ }^{3}$

\begin{abstract}
unitermos resumo
Necropsia

Diagnóstico clínico

Erros de diagnóstico

Objetivo: Analisar a concordância entre os diagnósticos clínicos e os achados anatomopatológicos obtidos por meio do exame necroscópico, a fim de determinar a freqüência com que a necropsia revela diagnósticos principais inesperados, que são relevantes para a causa do óbito. Métodos: Foram analisadas retrospectivamente 680 necropsias realizadas no Hospital de Base do Distrito Federal (HBDF), de janeiro de 1997 a dezembro de 2002. Para a comparação entre os achados clínicos e anatomopatológicos utilizou-se a classificação de Goldman, e fez-se a correlação segundo o sexo, a idade do paciente, a unidade de internação e o tempo de permanência hospitalar. Foram utilizados, como fonte de dados, os pedidos de necropsia, preenchidos pelos médicos-assistentes, e os laudos finais de necropsia. Resultados: A necropsia confirmou os diagnósticos principais formulados pela clínica em $69 \%$ das amostras. Em relação aos casos discordantes, $18 \%$ foram classificados como classe I (com potencial impacto na sobrevida do paciente) e $13 \%$ como classe II (sem o questionável impacto na sobrevida do paciente). Os principais diagnósticos discordantes mais freqüentes foram doenças do aparelho circulatório (acidente vascular cerebral, insuficiência cardíaca congestiva e miocardiopatia hipertrófica), moléstias infecciosas (meningite bacteriana, tuberculose miliar e neurotoxoplasmose) e neoplasias (linfomas). Conclusão: Evidenciou-se significativa discordância entre os diagnósticos clínicos e anatomopatológicos post mortem, mostrando que a necropsia ainda é um procedimento importante para o esclarecimento diagnóstico, bem como para a melhoria dos serviços de saúde.
\end{abstract}

Objective: To analyze the discrepancies between clinical and postmortem diagnoses in order to determine the frequency of relevant missed diagnoses detected at autopsy. Method: Six hundred and eighty patients autopsied at the Hospital de Base do Distrito Federal, Brazil, between January 1997 and December 2002 were retrospectively investigated. Agreement between clinical and postmortem findings was compared using Goldman's system. Medical records and final autopsy reports were reviewed. Results: Major clinical diagnoses were confirmed in $69 \%$ of analyzed autopsies. Eighteen percent of cases were classified as Coldman class I missed diagnosis (if known before death, might have led to prolonged survival) and $13 \%$ as class II (clinically missed major diagnosis for which survival would not have been changed). Most frequent missed major diagnoses were cardiovascular diseases (cerebral hemorrhagic infarction, congestive heart failure and hypertrophic cardiomyopathy), infectious diseases (purulent meningitis, miliar tuberculosis and neurotoxoplasmosis) and neoplasia (lymphoma). Conclusion; This study has found significant disagreement between clinical and postmortem examinations and reinforces the importance of autopsy in improving clinical diagnosis and in providing data that could be integrated in quality assurance programs. 


\section{Introdução}

Com o avanço da tecnologia diagnóstica complementar, o valor da necropsia, tanto para o ensino médico quanto para o aperfeiçoamento dos serviços de saúde, tem sido questionado. Os aspectos econômicos, a possibilidade de se revelar má prática médica e a falta de entusiasmo de médicos-assistentes e patologistas na realização e discussão de casos necropsiados são alguns dos fatores que podem ser considerados como desencorajadores para a realização desse procedimento ${ }^{(21,16)}$. Porém, a ênfase na utilização de modernos procedimentos diagnósticos tem sido apontada como um dos pontos mais relevantes ${ }^{(6,14)}$. Conseqüentemente, tem-se observado queda progressiva do número de necropsias realizadas em hospitais de todo o mundo. Nos EUA a taxa de necropsia (número de necropsias realizadas dividido pelo total de óbitos na instituição) que era de $50 \%$ em 1940, caiu progressivamente para 10\% a 15\% em 1985 e a menos de $5 \%$ na década de $1990^{(10,15,18)}$.

Seguindo a tendência mundial, as taxas de necropsia no Hospital de Base do Distrito Federal (HBDF) têm caído nos últimos anos. Foram realizadas, em média, 1.200 necropsias/ano de 1974 a 1976, e 208 necropsias/ano de 1999 a 2002, traduzindo uma redução de $82 \%$ do número absoluto de exames realizados. A taxa de necropsia nos anos de 1999 a 2002 foi de 14\%, em média.

A discordância entre os diagnósticos clínico e anatomopatológico post mortem tem sido discutida em numerosos relatos nas últimas décadas ${ }^{(1,3-6,8-10,12,13,16,17,19,20,22)}$. O estudo de Goldman et al. ${ }^{(6)}$ nas décadas de 1960, $1970 \mathrm{e}$ 1980 mostrou uma discordância de $22 \%$ nos diagnósticos principais, com impacto na sobrevida dos pacientes. Posteriormente, Shojania et al. ${ }^{(17)}$ e Roulson et al. ${ }^{(16)}$, em trabalhos de metanálise e revisão, mostraram resultados semelhantes, com discreta diminuição nas taxas de discordância desde a década de 1960 até a atual, apesar do grande progresso tecnológico na área médica.

Este estudo visa analisar a correlação entre os diagnósticos clínico e anatomopatológico obtidos por meio de exame necroscópico, a fim de determinar a freqüência com que a necropsia revela diagnósticos inesperados, que são relevantes para o conhecimento da causa do óbito.

\section{Métodos}

Foi realizado estudo transversal analítico para avaliação do grau de concordância entre os diagnósticos clínicos principais e os achados anatomopatológicos, em necrop- sias consecutivas realizadas no Núcleo de Citopatologia e Anatomia Patológica do Hospital de Base do Distrito Federal (NUCAP/HBDF), de janeiro de 1997 a dezembro de 2002.

O HBDF dispõe de 644 leitos, com uma média de 14 mil internações por ano, e ainda presta serviços assistenciais terciários em sistema hierarquizado que atende à população do DF e de outras localidades do país, atuando também como hospital de ensino. Ele conta com unidade de pronto-atendimento para urgências clínicas, cirúrgicas e politraumatizados. A unidade de tratamento intensivo (UTI) para adultos tem 21 leitos, a infantil seis e a neonatal dois leitos. Não há serviço de obstetrícia e a unidade de ginecologia atende a pacientes portadores de câncer do trato genital.

\section{Fonte de dados}

Utilizou-se fonte secundária de dados constituída pelos pedidos de necropsia e laudos finais de necropsia arquivados no NUCAP/HBDF. Os pedidos de necropsia foram preenchidos pelos médicos-assistentes e contêm:

- dados de identificação do paciente (nome, idade e sexo), unidade de atendimento e tempo de permanência hospitalar;

- história clínica resumida;

- exames complementares e a terapêutica aplicada;

- diagnósticos clínicos.

As necropsias no NUCAP/HBDF são realizadas por médicos-residentes em patologia e técnicos de necropsia sob supervisão de um patologista staff. Todas as necropsias são completas, sendo obtidos fragmentos de todos os órgãos para exame histopatológico.

Os laudos de necropsia são compostos de três partes:

- causa da morte, que se refere ao evento imediato da morte;

- diagnóstico principal (doença de base que levou ao óbito);

- diagnósticos secundários com aqueles achados de necropsia que podem ou não estar relacionados à doença principal e/ou ao óbito.

Toma-se como exemplo um caso de paciente com miocardiopatia chagásica, em insuficiência cardíaca congestiva (ICC), apresentando também bócio multinodular. O quadro 
evoluiu com dispnéia intensa e parada cardiorrespiratória não-responsiva às manobras de reanimação. A necropsia confirmou os diagnósticos clínicos e revelou tromboembolismo pulmonar recente e trombose venosa profunda. Nesse caso, a causa da morte foi tromboembolismo pulmonar, o diagnóstico morfológico principal (doença de base) foi miocardiopatia chagásica e os diagnósticos secundários foram trombose venosa profunda e bócio multinodular.

\section{Concordância entre os diagnósticos clínicos e os achados anatomopatológicos}

Para a avaliação da concordância entre os diagnósticos clínicos e os achados anatomopatológicos, utilizou-se a metodologia previamente descrita por Goldman et al.(6) composta de cinco classes:

- classe 1: diagnóstico principal discordante, com potencial impacto na sobrevida do paciente;

- classe 2: diagnóstico principal discordante, sem e/ou com questionável impacto na sobrevida do paciente;

- classe 3: diagnósticos secundários não formulados, mas que contribuíram para o óbito do paciente;

- classe 4: diagnósticos secundários não formulados, mas que não contribuíram para o óbito do paciente;

- classe 5: concordância completa entre as hipóteses de diagnóstico clínico e os achados anatomopatológicos.

Neste estudo considerou-se discordantes as classes 1 e 2 de Goldman, nas quais os diagnósticos principais não foram formulados antes do óbito. As demais classes 3, 4 e 5, nas quais os diagnósticos clínicos principais se confirmaram com os achados de necropsia, foram consideradas concordantes.

\section{Coleta dos dados}

Um questionário foi elaborado com os seguintes dados obtidos dos pedidos e relatórios finais de necropsia: nome, idade, sexo, unidade de atendimento, tempo de permanência hospitalar, diagnósticos clínicos, causa da morte, diagnósticos anatomopatológicos principais e secundários, e a classificação de Goldman, de acordo com o previamente descrito. $\mathrm{O}$ tempo de permanência hospitalar foi dividido em:

- até 1 dia de internação;

- entre dois e sete dias;

- entre oito e 14 dias;

- entre 15 e 28 dias;
- entre 29 e 60 dias;

- entre 61 e 90 dias;

- mais de 91 dias.

Todos os questionários foram revisados por dois médicos-residentes participantes do trabalho.

\section{Critérios de exclusão}

Foram excluídos os seguintes casos:

- pacientes que deram entrada no hospital em óbito ou aqueles cujo atendimento médico limitou-se a manobras de ressuscitação;

- casos nos quais os pedidos de necropsia estavam incompletos ou ilegíveis;

- aqueles em que os relatórios de necropsia estavam incompletos.

\section{Análise estatística}

A amostra foi selecionada de forma consecutiva simples e não-estratificada. Os dados foram categorizados em escala ordinal ou nominal e as taxas de concordância (número de casos concordantes dividido pelo total de casos) analisadas de forma pareada. Para testar as diferenças entre as proporções da taxa de concordância, utilizou-se o teste do qui-quadrado $\left(\chi^{2}\right)$ e, quando apropriado, o teste exato de Fisher, sendo considerada uma relevância estatística de $p<$ 0,05 . Para a realização dos cálculos estatísticos foi utilizado o programa BioEstat versão 4.0 (CT Brasil/IOEPA, 2005).

\section{Resultados}

Foram revistos 1.416 relatórios de necropsias consecutivas, sendo excluídos 151 casos $(10,6 \%)$ que chegaram em óbito ou cujo atendimento se limitou às manobras de reanimação. Outros 585 casos (41,3\%) foram excluídos por apresentarem relatórios incompletos ou ilegíveis. Os 680 casos restantes (48\%) foram incluídos no estudo.

\section{Concordância entre a hipótese de diagnóstico clínico e o diagnóstico anatomopatológico}

Entre os 680 casos analisados 470 (69\%) apresentaram concordância entre os diagnósticos principais: clínicos e anatomopatológicos. Concordância completa entre todos os diagnósticos clínicos e os achados da necropsia foi observada em 49\% (Figura 1). Os diagnósticos clínicos mais freqüentes confirmados pela necropsia foram - em 
ordem decrescente - cardiopatia congênita, síndrome da imunodeficiência adquirida (SIDA), cirrose, neoplasia do sistema nervoso central (SNC), infarto agudo do miocárdio (IAM) e cardiopatia reumática.

Entre os $31 \%$ de casos discordantes, os diagnósticos principais mais freqüentes formulados em vida e não confirmados à necropsia foram (em ordem decrescente) pneumonia $(8,2 \%)$, meningite bacteriana $(7,2 \%)$, acidente vascular cerebral hemorrágico (AVCH) $(3,8 \%)$ e doença isquêmica intestinal (3,7\%) (Tabela 1).

Diversas unidades mostraram elevada concordância, com a hematologia, as doenças infectoparasitárias e as cirurgias cardíacas, alcançando taxas em torno de $80 \%$, entretanto sem significância estatística. A pediatria alcançou taxa de concordância de $87 \%\left(\chi^{2} ; p=0,012\right)$ e a pneumologia de $43 \%\left(\chi^{2} ; p=0,036\right)$ (Figura 2).

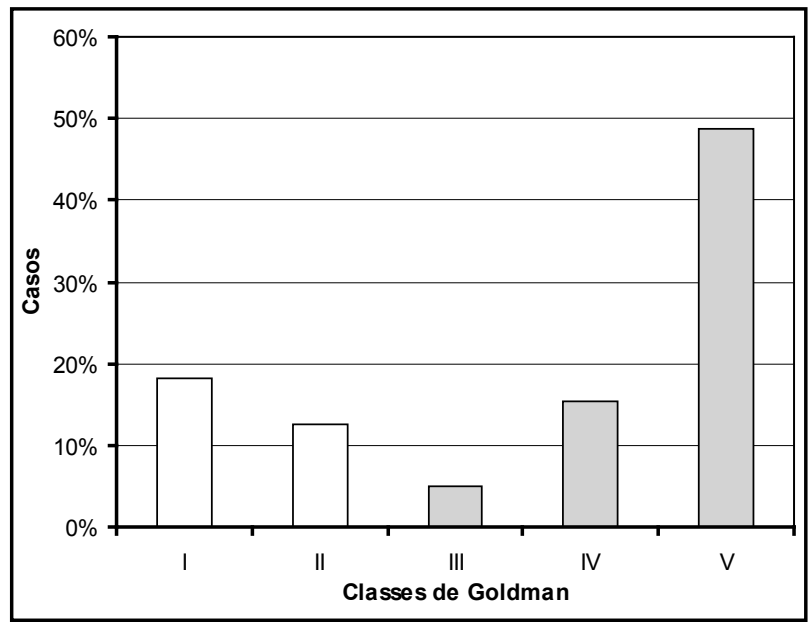

Figura 1 - Freqüência das classes de Goldman relacionadas a 680 pacientes avaliados no Núcleo de Citopatologia e Anatomia Patológica do Hospital de Base do Distrito Federal no período de 1997 a 2002
Principais diagnósticos discordantes nos 680 pacientes

Tabela 1 avaliados no HBDF de 1997 a 2002

- Diagnósticos

$\%$

Aparelho circulatório $\quad 24,4$

AVC

ICC

Miocardiopatia hipertrófica

Miocardiopatia chagásica

- Infecções

Meningite bacteriana

Tuberculose miliar

SIDA e neurotoxoplasmose

SIDA e doença de inclusão citomegálica

- Neoplasias primárias de:

Tecido linfóide (linfoma)

Fígado

Pâncreas

Pulmão

- Aparelho digestivo

Doença isquêmica intestinal

- Aparelho respiratório

Pneumonia

8,2

HBDF: Hospital de Base do Distrito Federal; AVC: acidente vascular cerebral; ICC: insuficiência cardíaca congestiva; SIDA: síndrome da imunodeficiência adquirida.

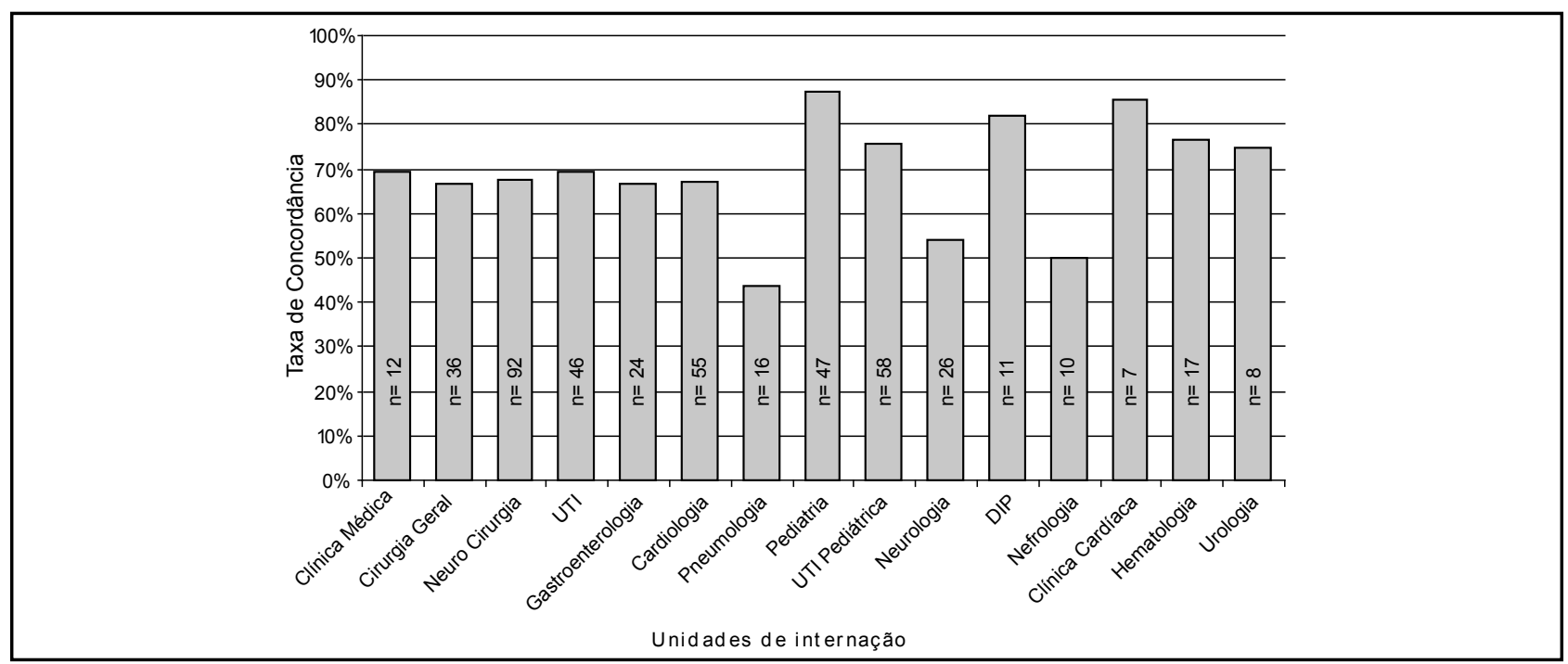

Figura 2 - Taxa de concordância de 680 pacientes internados no HBDF, por unidade de internação de 1997 a 2002 n: número de pacientes. 
Os diagnósticos principais comprovados pela necropsia em pacientes da pediatria foram: cardiopatia congênita (34\%), leucemia (4,9\%) e neoplasia do SNC (4,9\%).

Dos 16 casos necropsiados procedentes da pneumologia, apenas sete apresentaram concordância entre os diagnósticos clínicos e os achados anatomopatológicos. Oito de nove casos envolveram neoplasias primárias ou metastáticas para pulmão como diagnóstico clínico e/ou morfológico (Tabela 2).

\section{Taxa de concordância por faixa etária e sexo}

A taxa de concordância por faixa etária mostrou-se maior (95\%) no grupo de 2 a 12 anos, embora sem significância estatística $\left(\chi^{2} ; p=0,061\right)$. Nessa faixa etária, as principais hipóteses de diagnóstico clínico confirmadas pela necropsia foram cardiopatia congênita $(21,1 \%)$, síndrome de Reye (15,8\%), meningoencefalite viral (15,8\%), leucemia $(5,3 \%)$ e linfoma $(5,3 \%)$.

Em relação aos pacientes adultos (com mais de 20 anos), a taxa de concordância se manteve estável, com média de $68 \%$ ( $\pm 4 \%)$, e não mostrou variações estatisticamente significativas.
Não se observou diferença estatisticamente significativa $\left(\chi^{2} ; p=0,5\right)$ entre a taxa de concordância e o sexo dos pacientes.

Taxa de concordância por tempo de internação

A concordância entre os diagnósticos clínicos e de necropsia foi menor (62\%) em pacientes internados por até 1 dia antes do óbito, quando em comparação com os pacientes internados por períodos mais longos $\left(\chi^{2} ; p=0,02\right)$.

\section{Discussão}

O número de necropsias realizadas em hospitais tem

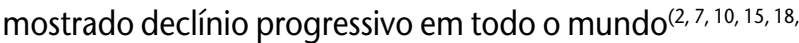
20, 22). No HBDF tem-se observado tendência semelhante. De 1974 a 1979 foram realizadas, em média, 1.027 necropsias por ano, em contraste com as 221/ano de 1994 a 2002.

Fatores econômicos, associados a grande demanda de biópsias, têm papel importante na redução do número de necropsias no HBDF. Contudo, também é notória a questão implícita de que a necropsia pouco acrescentaria ao diagnóstico clínico, na maioria dos casos, devido ao grande

\section{Comparação entre os diagnósticos principais, clínicos e anatomopatológicos, de pacientes}

Tabela 2 internados na unidade de pneumologia do HBDF de 1997 a 2002

\begin{tabular}{lll}
\hline Caso & Diagnóstico clínico & Diagnóstico anatomopatológico \\
\hline 1 & Neoplasia primária de pulmão & \\
2 & Neoplasia primária de pulmão & Carcinoma epidermóide de pulmão + EB \\
3 & Neoplaasia primária de pulmão & Tuberculose miliar \\
4 & Neoplasia primária de pulmão & Vasculite \\
5 & Neoplasia primária de pulmão & Mesotelioma fibroso maligno \\
6 & Pneumonia & Mieloma múltiplo \\
7 & Metástases sem sítio primário identificado & Metástases disseminadas sem sítio primário identificado \\
8 & Metástases sem sítio primário identificado & Carcinoma indiferenciado de tireóide \\
9 & DPOC & Pneumonia \\
10 & Neoplasia primária de pulmão & Adenocarcinoma de pulmão \\
11 & Neoplasia primária de pulmão & Carcinoma bronquioloalveolar de pulmão \\
12 & Neoplasia primária de pulmão & Carcinoma de pequenas células de pulmão \\
13 & Neoplasia primária de pulmão & Carcinoma epidermóide de pulmão \\
14 & DM & DM \\
15 & Neoplasia de ovário & Cistadenocarcinoma de ovário \\
16 & DPOC & DPOC \\
\hline
\end{tabular}

HBDF: Hospital de Base do Distrito Federal; EB: endocardite bacteriana; DPOC: doença pulmonar obstrutiva crônica; DM: diabetes mellitus. 
avanço tecnológico na medicina, sendo essa tendência uma das principais causas relatadas na literatura em todo o mundo ${ }^{(6,14)}$.

Das 1.416 necropsias revistas, 585 (41,3\%) foram excluídas por estarem com pedidos ou laudos de necropsia incompletos, uma situação semelhante à observada em outros relatos ${ }^{(1,5)}$

Dos 680 casos incluídos neste estudo, 18\% revelaram diagnósticos principais à autopsia, os quais, se realizados antes do óbito, poderiam resultar em diferente terapêutica e em maior sobrevida do paciente (classe I). Em outros 13\%, o diagnóstico principal, se confirmado, não teria repercussão na sobrevida do paciente (classe II). Tais resultados são comparáveis aos relatados no Brasil(1,5) e em outros

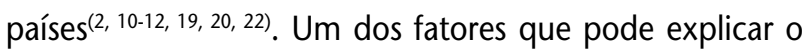
elevado número de necropsias que revelaram diagnóstico discordante (classes I e II) reside na própria característica do HBDF, ou seja, por ser integrante do Sistema Único de Saúde (SUS), ele presta serviços terciários, sendo um hospital de referência e de assistência a pacientes de difícil diagnóstico e tratamento. Além disso, a necropsia geralmente é solicitada ou realizada quando existem dúvidas sobre o diagnóstico clínico.

Vários estudos abordam o efeito potencial da queda do número de necropsias realizadas em hospitais, e sua possível relação com a elevada taxa de discordância diagnóstica, pois os casos com maior "certeza" diagnóstica não seriam examinados ${ }^{(19)}$. Essas necropsias poderiam elevar o número de casos concordantes. Alguns relatos mostram esse efeito, com taxas de discordância de 30\% em 1972 e de 14\% em 1992, em um centro com taxa de necropsia em torno de $90 \%{ }^{(16)}$. Em outros relatos, no entanto, essa "pré-seleção" de casos com maior dificuldade de cuidados assistenciais teve efeito relativamente pequeno em números de diagnósticos discordantes ${ }^{(6,13,18)}$.

Estudos realizados por Britton ${ }^{(3)}$ e Hartveit ${ }^{(8)}$ mostraram que a necropsia não alterou o diagnóstico clínico em 19\% a $25 \%$ dos casos em que os clínicos não tinham dúvidas quanto à causa do óbito, mas essa percentagem alcançou $35 \%$ a $45 \%$ nos casos em que apresentaram dúvidas.

Das 680 necropsias estudadas, 123 revelaram diagnósticos principais discordantes (classes I e II). Os principais achados anatomopatológicos nesses pacientes foram pneumonia (8,2\%), meningite bacteriana (7,2\%), $\operatorname{AVCH}(3,8 \%)$ e doença isquêmica intestinal $(3,7 \%)$ (Tabela 1 ). A pneumonia foi uma das principais causas de discordância diagnóstica referidas na literatura devido a sua variada apresentação clínica, podendo ser confundida com outras condições ${ }^{(16)}$. As unidades que exibiram maior número de casos discordantes foram a pneumologia, a neurologia e a nefrologia, porém somente a pneumologia $(p=0,036)$ mostrou resultados estatisticamente significativos. Oito dos nove casos discordantes da pneumologia envolveram neoplasia em pulmão como diagnóstico clínico ou anatomopatológico (Tabela 2). Outros estudos com metodologia semelhante mostraram que o câncer em pulmão representa difícil diagnóstico de correlação clinicopatológica, apresentando taxa de discordância de $44 \%{ }^{(12)}$. Além de ser sítio freqüente, tanto de metástases quanto de neoplasias malignas primárias, a apresentação dessa enfermidade pode simular outras condições pulmonares não-neoplásicas, como a tuberculose miliar apresentada por um de nossos pacientes.

A unidade de pediatria e o grupo etário de 2 a 12 anos mostraram maior taxa de concordância, em comparação com as demais unidades de internação (Figura 2) e faixas etárias. Nesses dois grupos, o diagnóstico clínico mais prevalente confirmado pela necropsia foi a cardiopatia congênita. Os portadores das mais variadas formas dessa doença tiveram o diagnóstico feito corretamente, sendo o óbito decorrente da impossibilidade de intervenção cirúrgica ou da descompensação cardiovascular. É interessante salientar que Chescheir e Reitnauer ${ }^{(4)}$, comparando os diagnósticos em vida com os diagnósticos de necropsias perinatais, mostraram que o estudo ultra-sonográfico é capaz de revelar aproximadamente $87 \%$ das anormalidades evidenciadas à autopsia.

A taxa de discordância entre os diagnósticos clínicos e anatomopatológicos post mortem em nosso hospital, semelhante à relatada na literatura ${ }^{(1,5,6,9,10,16-18)}$, reforça a importância da necropsia para o esclarecimento diagnóstico, contribuindo para aumentar o conhecimento médico do ambiente hospitalar e melhorar a qualidade da assistência prestada na instituição. No NUCAP/HBDF valoriza-se a necropsia através de reuniões semanais, entre a equipe de patologistas e residentes, com discussão dos achados macroscópicos das necropsias realizadas na semana, e reuniões anatomoclínicas quinzenais com as diferentes unidades hospitalares.

Embora os modernos métodos de investigação diagnóstica tenham representado um grande avanço para a medicina, a evidência de pequenas alterações nas taxas de discordância diagnóstica durante as últimas quatro décadas em todo o mundo merece reflexão. Valorizar a necropsia durante o ensino médico, favorecer maior aproximação entre clínicos e patologistas, agilizar a discussão dos resultados obtidos, possibilitando a análise sistematizada 
das causas de discordância entre os diagnósticos clínicos e anatomopatológicos, poderiam fazer com que essas informações sirvam realmente para a melhoria da qualidade da assistência médica prestada, indo além da prática da patologia, e servindo de base para a implantação de serviços assistenciais e avaliações epidemiológicas.

\section{Conclusão}

Este estudo evidenciou significativa discordância (31\%) entre os diagnósticos clínico e anatomopatológico post mortem, mostrando que a necropsia ainda é um procedimento importante para o esclarecimento diagnóstico e o aprimoramento dos serviços de saúde.

\section{Referências}

I. ALMEIDA, M. C. et al. Correlação diagnóstica anatomoclínica. Aferição retrospectiva do diagnóstico clínico em necropsias. Rev. Saúde Pública, v. 23, n. 4, p. 285-91 , 1989.

2. BRINKMANN, B.; DU CHESNE, A.; VENNEMANN, B. Recent data for frequency of autopsy in Germany (abstract). Dtsch Med Wochenschr, v. 127, p. 791-5, 2002

3. BRITTON, M. Clinical diagnostics: experience from 383 autopsied cases. Acta Med Scand, v. 196, p. 2 I I-9, 1974.

4. CHESCHEIR, N.C.; REITNAUER P.J. A comparative study of prenatal diagnosis and perinatal autopsy. J Ultrasound Med, v. 13, p. 45।-6, 1994.

5. CORADAZZI,A.L;; MORGANTI,A.L.C.; MONTENEGRO,M.R.G Discrepancies between clinical diagnoses and autopsy findings. Braz J Med Biol Res, v. 36, n. 3, p. 385-9I, 2003.

6. GOLDMAN, L. et al. The value of the autopsy in three medical eras. N Engl J Med, v. 308, p. 1000-5, 1983.

7. GRAVES, D. Is the autopsy dead? In: News from RCPA march 2004. Disponível em: http://www.rcpa.edu.au/applications/ DocumentLibraryManager2/upload/ls\%20the\%20Autopsy \%20Dead.pdf. Acesso em: I I jan. 2005.

8. HARTVEIT, F. Clinical and post-mortem assessment of the cause of death. J Pathol, v. 123, p. 193-210, 1977.

9. $\mathrm{KIRCH}, \mathrm{W}$.; SCHAFFI, C. Misdiagnosis at a university hospital in four medical eras. Medicine, v. 75, p. 29-40, 1996.

10. LANDEFELD C.S. et al. Diagnostic yield of the autopsy in a university hospital and a community hospital. N Engl J Med, v. 318, p. 1249-54, 1988.

I I. NEWTON, D. et al. How the pediatric autopsy yields valuable information in a vertically integrated health care system. Arch Pathol Lab Med, v. 128, p. 1239-46, 2004.

12 PERKINS, G.D. Discrepancies between clinical and postmortem diagnoses in critically ill patients: an observational study. Crit Care, v. 7. p. RI29-32, 2003.

13. PROBEGAR, M. et al. Should we confirm our clinical diagnostic certainty by autopsies? Intensive Care Med, v. 27, p. 1750-5, 2001 .

14. ROBERTS I.S. et al. Accuracy of magnetic resonance imaging in determining cause of sudden death in adults: comparison with conventional autopsy. Histopathology, v. 42, n. 5, p. 424-30, 2003.

I 5. ROBERTS,W.C.The autopsy: its decline and a suggestion for its revival. N Engl | Med, v. 299, p. 332-8, 1978.

16. ROULSON, J.; BENBOW, E.W.; HASLETON, P.S. Discrepancies between clinical and autopsy diagnosis and the value of post mortem histology: a meta-analysis and review. Histopathology v. 47, n. 6, p. 55।-9, 2005

17. SHOJANIA, K.G. et al. Changes in rates of autopsy-detected diagnostic errors over time: a systematic review. JAMA, v. 289, p. 2849-56, 2003

18. SHOJANIA, K.G. Archived cases and commentaries: pathology. The case: diagnostic surprises and the persistent value of the autopsy. Disponível em: http://www.webmm.ahrq.gov/cases. aspx?ic=54. Acesso em: I I jan. 2005.

19.SONDEREGGER-ISELI K. et al. Diagnostic errors in three medical eras: a necropsy study. Lancet, v. 355, p. 2027-31, 2000.

20. SPILLIOPOULOU, C. et al. Clinical diagnoses and autopsy findings: a retrospective analysis of 252 cases in Greece. Arch Pathol Lab Med, v. 129, p. 2 10-4, 2005.

21.STEIGMAN, C.K.The autopsy as a quality assurance tool: last rites or resurrection? Arch Pathol Lab Med, v. I20, p. 736-8, 1996.

22. TSE, G.M.K.; LEE, J.C.K. A 12-month review of autopsies performed at a university-affiliated teaching hospital in Hong Kong. Hong Kong Med J, v. 6, p. 190-4, 2000

\begin{tabular}{l|l} 
& Endereço para correspondência \\
\hline & Marcos Emanuel de Alcântara Segura \\
& Hospital de Base do Distrito Federal \\
& Núcleo de Citopatologia e Anatomia Patológica (NUCAP) \\
& SMHS, Quadra 101 - Asa Sul \\
& CEP 10330-150 - Brasília-DF \\
& e-mail: segura_m@terra.com.br
\end{tabular}

